Article type: Meta-analysis

\title{
Title: Periodontitis and circulating blood cells profiles: a systematic review and meta-analysis
}

João Botelho ${ }^{* 1,2}$, Vanessa Machado ${ }^{* 1,2}$, Syed Basit Hussain ${ }^{* 3}$, Syeda Ambreen Zehra ${ }^{4}$, Luís Proença ${ }^{5}$, Marco Orlandi ${ }^{3}$, José João Mendes ${ }^{2}$, Francesco D'Aiuto ${ }^{3}$

*Equally first author

${ }^{1}$ Periodontology Department, Clinical Research Unit (CRU), Centro de Investigação Interdisciplinar Egas Moniz (CiiEM), Instituto Universitário Egas Moniz (IUEM), Portugal

${ }^{2}$ Clinical Research Unit (CRU), CiiEM, IUEM, Portugal

${ }^{3}$ Periodontology Unit, University College London Eastman Dental Institute, London WC1X 8LD, UK

${ }^{4}$ Queen Mary, University of London

${ }^{5}$ Quantitative Methods for Health Research (MQIS), CiiEM, IUEM, Portugal

Running title: Hematologic changes \& Periodontitis

\section{Corresponding Author:}

João Botelho

Periodontology Department

Clinical Research Unit (CRU), Centro de Investigação Interdisciplinar Egas Moniz (CiiEM)

Instituto Universitário Egas Moniz, Egas Moniz Cooperativa de Ensino Superior

Campus Universitário, Quinta da Granja, 2829 - 511, Almada

Portugal

Phone: (+351) 212946737

Fax: not available

E-mail: ibotelho@egasmoniz.edu.pt

\section{CONFLICT OF INTEREST}

The authors declare no conflict of interest.

\section{ACKNOWLEDGEMENTS}

Nothing to declare 


\section{Summary}

Background: Periodontitis is a chronic inflammatory disease with local and systemic implications. Evidence suggests consistent hematologic changes associated with periodontitis. Our aim was to critically appraise the available evidence on hemogram, leukogram and thrombogram alterations in otherwise healthy patients suffering from periodontitis when compared to controls.

Methods: For this systematic review (SR), we searched MEDLINE, Web of Science, EMBASE and The Cochrane Library (CENTRAL) for studies published up to June 2020. Both observational and interventional studies with baseline standard hematologic levels were included. Outcomes of interest were baseline hemogram, leukogram and thrombogram values and the impact of periodontitis treatment on these outcomes. Upon risk of bias assessment, data extraction both qualitative and quantitative (standardised mean differences MD) analyses were performed. Random-effects meta-analyses were performed to provide pooled estimates. PRISMA guidelines were followed (PROSPERO reg: CRD42020164531).

Findings: A total of 45 studies, 8 intervention and 37 case-control, were identified after the final search of 3012 titles. Following quality assessment, 43 articles were deemed as of low risk of bias whilst two manuscripts with moderate risk. Meta-analyses confirmed that periodontitis was associated with both white and red cells lineages. Severe chronic periodontitis was associated with greater White Blood Cells count (WBC) (SMD of $0.53,95 \% \mathrm{Cl} 0.26-0.79$ ) when compared to controls. Patients with periodontitis were associated with higher number of neutrophils (MD of 7.16\%, 95\% Cl: 5.96-8.37) and lower Mean Platelet Volume (MD of $0.30 \mathrm{fL}, 95 \% \mathrm{Cl}: 0.49--0.10$ ) than healthy participants. Nonsurgical periodontal treatment (NSPT) was associated with a decrease in WBC levels (WMD of $0.2810 \% / \mathrm{L}, 95 \% \mathrm{Cl}-0.47-$ -0.08 ) in patients suffering from chronic periodontitis.

Interpretation: Periodontitis is associated with hematologic changes (SORT A recommendation). Higher WBC, neutrophils, erythrocyte sedimentation rate and lower MPV levels are the most common blood counts findings. The association between periodontitis and WBC could be causal in nature. Further research in assessing whether periodontitis causes circulating blood cells changes and identify the molecular mechanisms behind these associations are warranted. 


\section{Introduction}

Inflammation is a key host immune response triggered by a variety of factors including pathogens, damaged cells and toxic substances ${ }^{1}$. Cells and humoral changes are initiated by the host when a new inflammatory stimulus is identified. Monitoring body inflammation has over the years been possible thanks to a variety of cellular and circulating biomarkers ${ }^{2}$ among which, White Blood Cells count (WBC) and erythrocyte sedimentation rate (ESR) are considered both reliable and affordable hematologic surrogates.

When acute inflammation resolves and a chronic response ensues, important systemic implications can occur ${ }^{3,4}$. Non-communicable diseases including diabetes and cardiovascular diseases all recognize a common chronic inflammatory basis. Understanding all inflammatory triggering factors is urgent, which could in turn affect both the onset and progression of common chronic diseases as well their complications.

Periodontitis is a major chronic multifactorial inflammatory disease caused by a dysbiotic dental biofilm ${ }^{5}$ and characterized by a progressive destruction of the periodontium ${ }^{6}$. Periodontitis is one of the leading causes of tooth loss worldwide ${ }^{7,8}$. It is characterized by an exaggerated gingival inflammation with a distinctive inflammatory cell infiltrate and plethora of proinflammatory mediators which is not only confined to the gingival tissues but it also has systemic host changes ${ }^{9,10}$.

Systemic repercussions as a result of periodontitis have been comprehensively explored and revised 11,12 hence it is reasonable to hypothesize that such changes might be observed using hematologic surrogates. A recent systematic review linked periodontitis with a lower levels of red lineage cells parameters ${ }^{13}$ confirming the preliminary observational evidence that altered iron and ferritin levels are observed in patients with periodontitis when compared to controls ${ }^{14-18}$. Similarly patients with periodontitis appear to present with changes in WBC and platelets numbers ${ }^{18-21}$.

The aim of the present systematic review was to undertake a critical appraisal of the available evidence on hemogram, leukogram and thrombogram alterations in otherwise healthy patients suffering from periodontitis when compared to controls.

\section{Materials and Methods}

\section{Protocol and registration}

A protocol was registered in PROSPERO (ID: CRD42020164531) according to the Cochrane Handbook of Systematic Reviews of Interventions ${ }^{22}$ and it is hereby reported according to the PRISMA guidelines (Appendix S1) ${ }^{23}$.

\section{Focused questions and Eligibility criteria}

The following $\mathrm{PI}(\mathrm{E}) \mathrm{CO}$ questions were adopted:

1. "Do otherwise healthy patients suffering from periodontitis have altered hemogram, leukogram and thrombogram levels when compared to controls?".

2. "Do hemogram, leukogram and thrombogram levels decrease after non surgical periodontal treatment (NSPT)?"

The review followed these respective $\mathrm{PI}(\mathrm{E}) \mathrm{CO}$ statements:

1. Otherwise healthy patients (Patients - P); Periodontitis (Intervention/Exposure - E); No Periodontitis (Comparison - C); hemogram, leukogram and thrombogram levels (Outcome O) (detailed in Section Data extraction process and data items).

2. Otherwise healthy patients with periodontitis (Patients $-\mathrm{P}$ ); Non surgical periodontal treatment (Intervention/Exposure - E); Baseline levels of hemogram, leukogram and thrombogram levels 
(Comparison - C); post-treatment hemogram, leukogram and thrombogram levels (Outcome O) (detailed in Section Data extraction process and data items).

Studies were deemed eligible for inclusion based on the following criteria:

a) Observational (cross-sectional case-control) studies reporting hemogram, leukogram and thrombogram levels of periodontitis and not-periodontitis individuals;

b) Interventional studies (randomized controlled trials, [RCTs] and controlled clinical trials [CCTs]) reporting on hemogram, leukogram or thrombogram levels changes after periodontal treatment in patients with periodontitis;

c) Only studies including a clear periodontitis case definition;

d) Only studies with clear reporting of hematologic levels (hemogram, leukogram and thrombogram);

e) Studies only including patients with periodontitis but no other co-morbidities.

Interventional studies may be included to address the $\mathrm{PI}(\mathrm{E}) \mathrm{CO} 1$ if they provided data comparing periodontitis and non-periodontitis patients. Only interventional studies accounted to address $\mathrm{PI}(\mathrm{E}) \mathrm{CO}$ 2.

\section{Search strategy}

Detailed search strategies were conducted on the following databases: Pubmed, MEDLINE, CENTRAL [The Cochrane Central Register of Controlled Trials], Web of Science until June 2020. The following search syntax was used: "(periodontitis OR gingivitis OR periodontal health OR (periodontal diseases [MeSH])) AND (Hematologic Tests [MeSH] OR Blood Cell Count OR Erythrocyte Count OR Leukocyte Count OR Platelet Count OR Blood Coagulation Tests OR International Normalized Ratio OR Partial Thromboplastin Time OR Thrombelastography OR Prothrombin Time OR Thrombin Time OR Whole Blood Coagulation Time OR Blood Grouping and Crossmatching OR Blood Sedimentation OR Bone Marrow Examination OR Erythrocyte Aggregation OR Erythrocyte Indices OR Fibrin Clot Lysis Time OR FIGLU Test OR Hematocrit OR Hemoglobinometry OR Osmotic Fragility OR Platelet Function Tests OR Bleeding Time OR Clot Retraction OR Mean Platelet Volume OR Platelet Count OR Schilling Test OR ferritin)". No restrictions to publication year or language were applied. Grey literature was searched through appropriate databases and registers. Four periodontology journals were hand-searched (Journal of Clinical Periodontology, Journal of Periodontology, Periodontology 2000, Journal of Periodontal Research) to identify any additional articles. Authors were contacted when necessary to provide additional data and/or clarification on their reports.

\section{Study selection}

Study selection was assessed independently by two investigators (JB and VM), who performed the assessment of titles and/or abstracts of retrieved studies. Interexaminer reliability following full-text assessment was computed (kappa statistics) and any disagreements were resolved by discussion with a third author $(\mathrm{SBH})$.

\section{Data extraction process and data items}

Electronic data extraction included patients and study characteristics, first author's name, study design, publication year, country, inclusion and exclusion criteria, periodontitis case definition, mean age, the number of participants, gender, smoking habits, hematologic values. All data were independently extracted by two reviewers (VM and JB).

In this respect to consistency, the values were presented in the following unit of measures: WBC, Neutrophils, Lymphocytes, Monocytes, Eosinophils, Platelets as 10\%/L; the percentage of Neutrophils, 
Lymphocytes, Haematocrit and Red Cell Distribution Width (RDW); RBC as $10 \% / \mathrm{mcL}$; Mean Corpuscular Volume (MCV) and Mean Platelet Volume (MPV) as $\mathrm{fL}$; Haemoglobin and Mean Corpuscular Haemoglobin Concentration (MCHC) as $\mathrm{g} / \mathrm{dL}$; Mean Corpuscular Haemoglobin (MCH) as $\mathrm{pg}$; ESR as $\mathrm{mm} / \mathrm{h}$; Serum Iron $(\mu \mathrm{g} / \mathrm{dl})$ and Ferritin $(\mathrm{ng} / \mathrm{ml})$.

Regarding the exposure, we found multiple case definitions for periodontitis. A lack of consistency on the case definitions hampered the assessment and interpretation of data. Studies were sub-grouped according to: 1) a confident or non-confident case definition of periodontitis based on previously reported criteria ${ }^{24}$ and 2) reporting established periodontitis case-definitions (European Federation of Periodontology and/or American Academy of Periodontology) other case definitions-

Confident case definitions of periodontitis were as follows: generalized chronic periodontitis (at least $30 \%$ sites with clinical attachment loss $(C A L) \geq 4 \mathrm{~mm}$ ) ${ }^{25}$; at least two sites on different teeth with CAL $6 \mathrm{~mm}$ and at least one site with probing pocket depth (PPD) $4 \mathrm{~mm}$ (CDC/AAP periodontitis definition) ${ }^{26}$; interdental CAL is detectable at $\geq 2$ non-adjacent teeth, or buccal or oral CAL $\geq 3 \mathrm{~mm}$ with pocketing $>3$ $\mathrm{mm}$ is detectable at $\geq 2$ teeth ${ }^{27}$; at least five sites with $C A L \geq 6 \mathrm{~mm}$. For non-confident case definitions, the following reported criteria were considered: community periodontal index of treatment need (CPITN) score $3 / 4$ in at least one quadrant; 'Alveolar bone loss' without other measurements of PPD/ CAL; unclear diagnostic criteria for periodontitis.

\section{Risk of bias (RoB)}

The Newcastle-Ottawa (NOS) Scale for case-control and cohort studies was used by one calibrated reviewer appraising evidence from case-control and cross-sectional studies (SBH). For calibration purposes, a random sample of 10 studies was assessed and reassessed 2 days later (agreement of 0.96). Studies with 7-9 stars were deemed as of low RoB, studies with 5-6 stars of moderate RoB, whilst studies with less than 5 stars were deemed of high RoB. Any dispute was resolved by discussion with a second author (JB). For interventional studies, the ROBINS-I tool (Risk Of Bias In Non-randomized Studies - of Interventions) ${ }^{28}$.

\section{Quantitative analyses}

For continuous data, mean values and standard deviations (SD) were used and analysed with standard mean differences (MD) and 95\% confidence intervals (Cls). The unit of measurements were standardized and converted appropriately as above mentioned. If median and interquartile range were reported in the selected studies, mean and SD were calculated using a previously reported formula 29. In the present analysis, reported data were pooled, irrespective of the individual case definition. Subgroup meta-analysis estimates were pooled using DerSimonian-Laird random-effects strategy ${ }^{30}$.

We used the $\mathrm{R}$ version 3.4.1 to calculate estimates through DerSimonian-Laird random-effects model ${ }^{30}$, as previously described ${ }^{31}$. All random-effects meta-analysis and forest plots were performed using 'meta' package ${ }^{31}$. Using $\mathrm{I}^{2}$ index and Cochrane's $Q$ statistic we inspected statistical heterogeneity $(p<0.1)$, and $x 2$ test calculated overall homogeneity ${ }^{22}$. All tests were two-tailed, with alpha set at 0.05 . Further, forest plots provide the weight percentage given to each study. For meta-analysis including 10 or more studies, we analysed publication bias ${ }^{22}$. Overall ES estimates were reported with $95 \% \mathrm{Cl}$.

We have conducted a first sensitivity analysis to explore the influence of using a MD approach (which allows clinical interpretation of data) versus employing a ratio of means (RoM) approach (permits direct interpretation as a percent difference between cases and controls and attenuates eventual heterogeneity withing the estimates) ${ }^{32-35}$.

Then, a priori sensitivity analyses were defined when studies with moderate and high RoB were examined in the pooled estimates, as well meta-regression was performed towards the influence of smokers present in the sample (through the ratio of smokers Periodontitis/Non-Periodontitis) and the 
gender ratio (M/W ratio). Further, studies separating non-severe from severe periodontitis cases were used to compare blood levels and hence to infer the influence of severity.

We employed the Strength of Recommendation Taxonomy (SORT) to appraise the strength and quality of the evidence ${ }^{36}$.

\section{Results \\ Study selection}

The search strategy retrieved 6107 possibly relevant publications. After duplicates removal, 3197 manuscripts were examiner and 2860 were excluded after titles and/or abstracts review. Out of the 158 remaining articles assessed for full text search/eligibility, 114 articles were further excluded (Appendix S2). A total of 37 case-control studies and 8 intervention trials were included for qualitative and quantitative synthesis (Figure 1). Good inter-examiner reliability at the full-text screening was recorded (kappa score $=0.915,95 \% \mathrm{Cl}: 0.929-0.943$ ).

\section{Studies characteristics}

The systematic review identified 37 case-control studies ${ }^{14-16,18-21,37-66}$ and 8 intervention studies 67-74 from fifteen different countries, across Asia, Europe and America (Appendices S3). A total of 11,368 participants were included in this review. Quantitative observational analyses included 4,228 healthy individuals and a total of 3,329 patients with periodontitis (subcategorised as 1,920 suffering from chronic and 1,449 from aggressive form). In the comparison of delta values of WBC (before and after periodontal treatment), a total of 254 patients from 8 intervention trials were included. When assessing the impact of severity of periodontitis on hematologic changes, 2878 patients were included in total, 1820 with non-severe CP and 1058 with severe. In three studies $38,57,66$ using a specific periodontitis case definition (CPITN) healthy and mild periodontitis cases were grouped as a single category, hence in our analyses we included patients with moderate or severe periodontitis compared with the rest.

Thirty-five studies presented with low RoB (22 with 9/9, 10 with 8/9 and 3 with 7/9 scores) (Appendix S4). Only two manuscripts presented moderate risk of bias with an overall score of $6 / 9$. The main reason for bias arose from the representativeness of the cases. The majority of articles adopted an adequate periodontitis case definition $(97.3 \%, n=36)$ and control participants $(91.9 \%, n=34)$ whilst a considerable number of studies failed to include representative samples $(46.1 \%, n=13)$. When assessing comparability of cases and controls of design or analysis, ascertainment of exposure, method of ascertainment for cases and controls and non-response rate, all article presented low RoB $(100.0 \%$, $\mathrm{n}=43$ ). Eight intervention trials presented low risk of bias according to the ROBINS-I tool (Appendix S5).

\section{Effect of Periodontitis and its treatment on Circulating Blood Cells}

Firstly, RoM approach (versus MD approach) presented no potential added-value at least in terms of heterogeneity and inference as confirmed in the first sensitivity tests for periodontitis, chronic periodontitis and aggressive periodontitis (Appendix S6). Hence, we proceeded with interpretation of the estimates provided by the MD approach.

In our analysis, periodontitis was associated with an increased count of white cells (SMD of $0.89,95 \% \mathrm{Cl}$ $0.72-1.05$ ) (Figure 2) and Neutrophils (SMD of $0.76,95 \% \mathrm{Cl} 0.57-0.95$ ) (Table 1) and high level of heterogeneity ( $75.4 \%$ and $72.1 \%$, respectively) (Appendices S7 and S9). When assessing the red cells panel, patients with periodontitis exhibited reduced RBC, haemoglobin, haematocrit, MCV, MCH surrogates, but not ESR that was elevated (Table 1). There were no differences in the thrombogram panel. 
We then analysed separately according to the type of periodontitis. Chronic periodontitis was associated with an average higher count of white cells (SMD of 1.01,95\% Cl $0.73-1.29)$ and Neutrophils (SMD of $0.80,95 \% \mathrm{Cl} 0.47-1.13$ ) and high level of heterogeneity ( $80.3 \%$ and $76.5 \%$, respectively) (Appendices S7-S17). Further, patients with chronic periodontitis exhibited reduced RBC, haemoglobin, haematocrit, $\mathrm{MCH}, \mathrm{MCHC}$ levels. In particular, periodontitis was linked to reduced Haematocrit (MD of $-0.39 \%, 95 \%$ $\mathrm{Cl}-0.58--0.20$ ) and higher ESR (MD of $2.30 \mathrm{~mm} / \mathrm{h}, 95 \% \mathrm{Cl} 1.25-3.36$ ) values when compared to healthy individuals (Appendices S18-S32). No differences in platelet counts were reported (Appendix S33).

When patients with aggressive periodontitis were analysed, similar results were observed: increased WBC (SMD of $0.6010^{9} / \mathrm{L}, 95 \% \mathrm{Cl} 0.50-0.69$ ) and Neutrophils counts (SMD of $0.7510^{9} / \mathrm{L}, 95 \% \mathrm{Cl} 0.52-$ 0.97) (Appendices S7-S17). Similar red cells lineages profiles were observed in patients with aggressive periodontitis when compared to healthy controls (decreased haematocrit, MCV and MCH levels) (Appendices S18-S32).

Lastly when thrombogram panels were compared, patients with aggressive periodontitis were associated with lower MPV values (SMD of $-0.30 \mathrm{fL}, 95 \% \mathrm{Cl}-0.49--0.10$ ) when compared to healthy controls (Appendices S35).

In the secondary analysis of hematologic changes within patients with periodontitis and according to disease severity, the meta-analysis confirmed that patients with severe periodontitis had higher WBC (SMD of $0.53,95 \% \mathrm{Cl} 0.26-0.79$ ) and in particular Neutrophils (SMD of $0.40,95 \% \mathrm{Cl} 0.38-0.42$ ) when compared to cases with moderate disease (Appendix S36-38).

Lastly, when intervention trials were appraised, nonsurgical periodontal therapy was associated with a consistent decrease in WBC (WMD of $-0.3910 \% / \mathrm{L}, 95 \% \mathrm{Cl}-0.65--0.12$ ) (Figure 3, Table 4 and Appendices S39).

Publication bias was assessed in all meta-analyses (Tables 1-3). Overall articles reporting on platelets changes were affected by publication bias $(p=0.009)$ as well as neutrophils when assessed in patients with chronic periodontitis $(p=0.004)$. Meta-regression analysis confirmed the influence of $M / W$ ratio on WBC (Estimate $=0.5, p=0.0112$ ), but not for $R B C$ (Estimate $=-0.17, p=0.1896$ ) and platelet counts (Estimate=24.29, $\mathrm{p}=0.0982$ ). Also, the presence of smokers had no significant influence for WBC (Estimate $=0.22, p=0.4229$ ), RBC (Estimate $=0.10, p=0.2492$ ) and Platelet levels (Estimate $=3.76$, $\mathrm{p}=0.8637$ ).

Lastly meta-analyses of studies according to the case definitions of periodontitis threshold were performed (Appendix S40). The assessment revealed potential sources of bias particularly the suitability of case definition for cases and controls. Non-confident case definitions of periodontitis confirmed to be a source of variability and heterogeneity when compared with confident definitions. Analysis using confident case definitions from the EFP and/or AAP resulted in lower heterogeneous results.

Using the SORT recommendation we concluded that periodontitis is strongly associated with hematologic changes (SORT A) ${ }^{36}$.

\section{Discussion}

This systematic review supports a consistent association of periodontitis with hematologic changes. The quantitative analysis provided by observational studies revealed that periodontitis is linked to higher WBC (with particular increase of neutrophils) and a decrease of some erythrocyte and platelet lineages. When interventional evidence was appraised, non surgical periodontal treatment was associated with a decrease in WBC.

Our results are consistent with previous reviews although previous reports were aimed at assessing only indirectly the impact of periodontitis on circulating white cells ${ }^{13,75-77}$. In addition to this, the present 
review included considerably higher number of studies, focused on more blood cells and reports on outcome changes before and after periodontal treatment, which may be seen as novel.

The increase of WBC, Neutrophils and ESR observed in this review portrays the inflammatory response of the body to an infection of periodontal cause, endorsing the role of periodontitis as an inducer of inflammation. These surrogates are widely accepted markers of systemic inflammation in cardiovascular disease ${ }^{78}$ and diabetic patients ${ }^{79}$. Elevated WBC counts were found to be a risk factor for coronary heart disease, ischemic stroke incidence and cardiovascular disease mortality in Americans ${ }^{80}$. Elevated WBC levels and chronic inflammation may also contribute to the development of macro- and microvascular complications in diabetes ${ }^{81}$. Inflammation is therefore consistently established as key to the development and complications of cardiovascular diseases and diabetes 82,83. Comprehensively, the inflammatory response towards periodontal infection acquires particularly relevance in systemically undermined patients with non-communicable diseases because the enduring of a chronic low-grade inflammation might put these particular patients at risk, namely endothelial dysfunction ${ }^{84-86}$, stroke events ${ }^{87,88}$, or even uncontrolled glycemia in diabetic patients ${ }^{89}$.

A variety of mechanisms could explain the association between periodontitis and increased WBC and neutrophils. The gingival inflammation response is characterised by a dense inflammatory infiltrate rich in leucocytes which could in turn be damped into the systemic circulation ${ }^{90-92}$. Alternatively, the local continuous inflammatory and bacterial interplay could stimulate the bone marrow in producing chronically higher number of inflammatory cells ${ }^{93}$. Further, periodontal bacteria could invade the periodontal tissues via the ulcerated epithelium, and trigger a systemic response to counteract any detrimental effect.

Further confirmation of a possible causal link between periodontitis and leucocytes count comes from the evidence appraised on intervention studies pointing to a consistent reduction of WBC after basic periodontal treatment. Treatment of periodontitis which results in resolution of the local inflammatory response might also alleviate the chronic cellular inflammatory changes. However, these results should be still interpreted with caution because of the limited sample size and methodological discrepancies of clinical studies included.

In this review we also attempted to provide estimates of association between periodontitis and hematologic changes using older case definitions of periodontitis (chronic vs aggressive forms). This review confirmed that both forms of disease were associated consistently with similar hematologic changes. This is in contrast with the past efforts suggesting that the aggressive forms of periodontitis did present with distinct inflammatory cells profiles ${ }^{90}$, mainly focusing on the function of neutrophils. Altered chemotaxis or phagocytosis had been advocated as plausible mechanisms linked to destruction of the periodontal tissues ${ }^{90}$. This apparent contradiction is based on the fact that our review only focused on the number of cells rather their function. Further studies should be implemented to investigate the specific functional patterns of inflammatory cells observed in periodontitis patients when compared to controls.

This review revealed that diagnosis of periodontitis was also associated with the decrease of red cells lineages, in agreement with recent reviews ${ }^{13,77}$. Nevertheless, both previous reviews focused mainly on studies including only male participants. It is well established the notion that chronic inflammation affecting negatively red cell numbers and morphology. This is elsewhere referred to anaemia of chronic inflammation ${ }^{94-96}$. The systemic inflammatory process subsequent to the gingival bacterial burden could impact on the processes of erythropoiesis and red cells life span. This type of anaemia also displays impaired iron trafficking and iron availability ${ }^{97}$, though our results do not support this association possibly due to the shortage of available studies ${ }^{77}$.

Lastly when assessing the impact of periodontitis on platelet lineages, a recent study reported increased platelet count in severe chronic periodontitis cases ${ }^{98}$. Our review did not confirm consistent platelet alterations although MPV was increased in patients with aggressive periodontitis when compared to healthy individuals. A reactive thrombocytosis subsequent to increased hepatic thrombopoietin could represent one of the mechanisms explaining these associations ${ }^{99-101}$. Interestingly, platelets share a 
common differentiation pathway with WBC ${ }^{102-104}$ and the same mechanism explaining higher leucocytes numbers could be responsible of low platelets. Nevertheless, considering the low number of studies that were used to explore this analysis, future studies are warranted to clarify this association.

\section{Strengths and Potential Limitations}

Despite the comprehensive approach undertaken in this review to appraise all evidence linking periodontitis and hematologic changes, some limitations should be reported. Firstly, the high level of heterogeneity observed in some of the reported estimates limits the validity and generalizability of quantitative analyses. Nevertheless, sensitivity analysis confirmed that even a RoM approach did not attenuate heterogeneity of the estimates. The lack of consistency on the periodontitis case definition did not allow for more robust analyses of the extent and severity of periodontitis with all blood hallmarks. Sensitivity analyses were therefore performed confirming the interpretation of results from the main meta-analyses. Future studies should include up-to-date case definitions as well as more detailed data concerning the extent and severity of periodontitis ${ }^{27}$.

Second, we should point out that evidence from observational studies included in this review would only inform on the nature of the association between periodontitis and hematologic changes without confirming causality. The analysis of intervention studies however confirmed a possible reversible effect of periodontitis on WBC/neutrophils. Even considering the fact that the multiplicity of intervention types in the included NRSI may contribute to increase the level of bias, this fact did not prevent the results obtained having been completely homogeneous. Notwithstanding, we should carefully interpret this result, since the comparison derived from baseline versus post-intervention data. Hence, randomized controlled trials are warranted to confirm this result in a more consistent design methodology.

Publication bias was detected on platelets and neutrophils changes in chronic periodontitis affecting the quality of evidence and, therefore, we must interpret carefully the results for these markers.

\section{Conclusion}

Periodontitis increases WBC counts and periodontal treatment mitigates this effect. This is consistent with the notion that periodontitis causes a reversible state of systemic inflammation. 
Contributors: JB, VM and SH all steps of protocol, search, inclusion. JB and LP did the statistical analysis. JB, VM and BH wrote the first draft of the report with input from FD. All authors wrote the final draft, read and approve the final manuscript.

Funding: Francesco D'Aiuto received a BRC's Oral Health and Disease grant.

Competing interests: We declare no competing interests. 


\section{References}

1 Chen L, Deng $\mathrm{H}$, Cui $\mathrm{H}$, et al. Oncotarget 7204 www.impactjournals.com/oncotarget Inflammatory responses and inflammation-associated diseases in organs. Oncotarget 2018; 9: 7204-18.

2 Watson J, De Salis I, Salisbury C, Hamilton W. 'I'm fishing really'-Inflammatory marker testing in primary care: A qualitative study. Br J Gen Pract 2016; 66: e200-6.

3 Furman D, Campisi J, Verdin E, et al. Chronic inflammation in the etiology of disease across the life span. Nat Med 2019; 25: 1822-32.

4 Franceschi C, Garagnani P, Parini P, Giuliani C, Santoro A. Inflammaging: a new immunemetabolic viewpoint for age-related diseases. Nat Rev Endocrinol 2018; 14: 576-90.

Roberts FA, Darveau RP. Microbial protection and virulence in periodontal tissue as a function of polymicrobial communities: Symbiosis and dysbiosis. Periodontol 2000 2015; 69: 18-27.

Papapanou PN, Sanz M, Buduneli N, et al. Periodontitis: Consensus report of workgroup 2 of the 2017 World Workshop on the Classification of Periodontal and Peri-Implant Diseases and Conditions. In: Journal of Clinical Periodontology. 2018: S162-70.

Petersen PE, Ogawa $\mathrm{H}$. The global burden of periodontal disease: Towards integration with chronic disease prevention and control. Periodontol 2000 2012; 60: 15-39.

Kassebaum NJ, Bernabé E, Dahiya M, Bhandari B, Murray CJL, Marcenes W. Global burden of severe periodontitis in 1990-2010: A systematic review and meta-regression. J Dent Res 2014; 93: 1045-53.

Ebersole JL, Dawson D, Emecen-Huja P, et al. The periodontal war: microbes and immunity. Periodontol 2000 2017; 75: 52-115.

10 Ebersole JL, Graves CL, Gonzalez OA, et al. Aging, inflammation, immunity and periodontal disease. Periodontol 2000 2016; 72: 54-75.

11 Jepsen S, Caton JG, Albandar JM, et al. Periodontal manifestations of systemic diseases and developmental and acquired conditions: Consensus report of workgroup 3 of the 2017 World Workshop on the Classification of Periodontal and Peri-Implant Diseases and Conditions. $J$ Clin Periodontol 2018; 45: S219-29.

12 Chan S, Pasternak GM, West MJ. The place of periodontal examination and referral in general medicine. Periodontol 2000 2017; 74: 194-9.

13 França LF de C, da Silva FRP, di Lenardo D, et al. Comparative analysis of blood parameters of the erythrocyte lineage between patients with chronic periodontitis and healthy patients: Results obtained from a meta-analysis. Arch Oral Biol 2019; 97: 144-9.

14 Chakraborty S, Tewari S, Sharma RK, Narula SC. Effect of Non-Surgical Periodontal Therapy on Serum Ferritin Levels: An Interventional Study. J Periodontol 2014; 85: 688-96.

15 Latha S, Thirugnanamsambandan S, Arun R, Masthan KMK, Malathi L, Rajesh E. Serum ferritin level and red blood cell parameters in healthy controls and chronic periodontitis patients. J Pharm Bioallied Sci 2015; 7: S184-9.

16 Guo LN, Yang YZ, Feng YZ. Serum and salivary ferritin and Hepcidin levels in patients with chronic periodontitis and type 2 diabetes mellitus. BMC Oral Health 2018; 18: 1-9.

17 Leite FRM, Nascimento GG, Scheutz F, López R. Effect of Smoking on Periodontitis: A Systematic Review and Meta-regression. Am. J. Prev. Med. 2018; 54: 831-41.

18 Nibali L, Darbar U, Rakmanee T, Donos N. Anemia of inflammation associated with periodontitis: Analysis of two clinical studies. J Periodontol 2019; 90: 1252-9.

19 Temelli B, Yetkin Ay Z, Aksoy F, et al. Platelet indices (Mean platelet volume and platelet distribution width) have correlations with periodontal inflamed surface area in coronary artery disease patients: A pilot study. J Periodontol 2018; 89: 1203-12.

20 Zhan $\mathrm{Y}$, Lu R, Meng H, Wang X, Sun X, Hou J. The role of platelets in inflammatory immune responses in generalized aggressive periodontitis. J Clin Periodontol 2017; 44: 150-7. 
Anand PS, Sagar DK, Mishra S, Narang S, Kamath KP, Anil S. Total and differential leukocyte counts in the peripheral blood of patients with generalised aggressive periodontitis. Oral Heal Prev Dent 2016; 14: 443-50.

Higgins J, Green S. Cochrane Handbook for Systematic Reviews of Interventions. 5.1.0 (updated March 2011). The Cochrane Collaboration. (https://www.cochrane-handbook.org)., 2011.

Liberati A, Altman DG, Tetzlaff J, et al. The PRISMA statement for reporting systematic reviews and meta-analyses of studies that evaluate health care interventions: Explanation and elaboration. PLoS Med 2009; 6. DOI:10.1371/journal.pmed.1000100.

24 Muñoz Aguilera E, Suvan J, Buti J, et al. Periodontitis is associated with hypertension: A systematic review and meta-analysis. Cardiovasc Res 2020; 116: 28-39.

Armitage G. Development of a Classification System for Periodontal Diseases and Conditions. Ann Periodontol 1999; 4: 1-6.

26 Eke PI, Page RC, Wei L, Thornton-Evans G, Genco RJ. Update of the Case Definitions for Population-Based Surveillance of Periodontitis. J Periodontol 2012; 83: 1449-54.

Tonetti MS, Greenwell H, Kornman KS. Staging and grading of periodontitis: Framework and proposal of a new classification and case definition. J Periodontol 2018; 45: S149-61.

Sterne JA, Hernán MA, Reeves BC, et al. ROBINS-I: A tool for assessing risk of bias in nonrandomised studies of interventions. BMJ 2016; 355: 4-10.

Hozo SP, Djulbegovic B, Hozo I. Estimating the mean and variance from the median, range, and the size of a sample. BMC Med Res Methodol 2005; 5: 1-10.

30 Schwarzer G, Carpenter JR, Rücker G. Meta-Analysis with R. Springer, 2015.

31 Schwarzer G. meta: An R Package for Meta-Analysis. R News 2007; 7: 40-5.

32 Friedrich JO, Adhikari NKJ, Beyene J. The ratio of means method as an alternative to mean differences for analyzing continuous outcome variables in meta-analysis: A simulation study. BMC Med Res Methodol 2008; 8. DOI:10.1186/1471-2288-8-32.

33 Friedrich JO, Adhikari NKJ, Beyene J. Ratio of geometric means to analyze continuous outcomes in meta-analysis: Comparison to mean differences and ratio of arithmetic means using empiric data and simulation. Stat Med 2012; 31: 1857-86.

34 Higgins J, White I, Anzures-Cabrera J. Meta-analysis of skewed data: Combining results reported on log-transformed or rawscales. Stat Med 2008; 28: 6072-92.

35 Friedrich JO, Adhikari NKJ, Beyene J. Ratio of means for analyzing continuous outcomes in meta-analysis performed as well as mean difference methods. $J$ Clin Epidemiol 2011; 64: 55664.

36 Newman MG, Weyant R, Hujoel P. JEBDP Improves Grading System and Adopts Strength of Recommendation Taxonomy Grading (SORT) for Guidelines and Systematic Reviews. J Evid Based Dent Pract 2007; 7: 147-50.

37 Zhan $\mathrm{Y}$, Lu R, Meng $\mathrm{H}$, Wang $\mathrm{X}$, Hou J. Platelet activation and platelet-leukocyte interaction in generalized aggressive periodontitis. J Leukoc Biol 2016; 100: 1155-66.

38 Zimmermann $\mathrm{H}$, Hagenfeld $\mathrm{D}$, Diercke $\mathrm{K}$, et al. Pocket depth and bleeding on probing and their associations with dental, lifestyle, socioeconomic and blood variables: A cross-sectional, multicenter feasibility study of the German National Cohort. BMC Oral Health 2015; 15: 1-9.

39 Sharma A, Astekar M, Metgud R, Soni A, Verma M, Patel S. A study of C-reactive protein, lipid metabolism and peripheral blood to identify a link between periodontitis and cardiovascular disease. Biotech Histochem 2014; 89: 577-82.

40 Kumar BP, Khaitan T, Ramaswamy P, Sreenivasulu P, Uday G, Velugubantla RG. Association of chronic periodontitis with white blood cell and platelet count - A Case Control Study. J Clin Exp Dent 2014; 6: 214-7.

41 Pereira LC, Nascimento JCR, Rêgo JMC, et al. Apolipoprotein E, periodontal disease and the risk for atherosclerosis: a review. Arch Oral Biol 2019; 98: 204-12. 
Anumolu VSH, Srikanth A, Paidi K. Evaluation of the relation between anemia and periodontitis by estimation of blood parameters: A cross-sectional study. J Indian Soc Periodontol 2016. DOI:10.4103/0972-124X.176392.

Gani DK, Mallineni SK, Ambalavanan, Ramakrishnan, Deepalakshmi, Emmadi P. Estimation of the levels of C-reactive protein, interleukin-6, total leukocyte count, and differential count in peripheral blood smear of patients with chronic periodontitis in a South Indian population. West Indian Med J 2012; 61: 826-31.

44 Gaddale R, Mudda JA, Karthikeyan I, Desai SR, Shinde H, Deshpande P. Changes in cellular and molecular components of peripheral blood in patients with generalized aggressive periodontitis. J Investig Clin Dent 2016; 7: 59-64.

45 Anand PS, Sagar DK, Ashok S, Kamath KP. Association of aggressive periodontitis with reduced erythrocyte counts and reduced hemoglobin levels. J Periodontal Res 2014; 49: 71928.

Pejčić A, Kesić L, Pešić Z, Mirković D, Stojanović M. White blood cell count in different stages of chronic periodontitis. Acta Clin Croat 2011; 50: 159-67.

Monteiro AM, Jardini MAN, Alves S, et al. Cardiovascular Disease Parameters in Periodontitis. J Periodontol 2009; 80: 378-88.

48 Papapanagiotou D, Nicu EA, Bizzarro S, et al. Periodontitis is associated with platelet activation. Atherosclerosis 2009; 202: 605-11.

49 Shi D, Meng H, Xu L, et al. Systemic Inflammation Markers in Patients With Aggressive Periodontitis: A Pilot Study. J Periodontol 2008; 79: 2340-6.

50 Bizzarro S, Van Der Velden U, Ten Heggeler JMAG, et al. Periodontitis is characterized by elevated PAI-1 activity. J Clin Periodontol 2007; 34: 574-80.

51 Havemose-Poulsen A, Westergaard J, Stoltze K, et al. Periodontal and Hematological Characteristics Associated With Aggressive Periodontitis, Juvenile Idiopathic Arthritis, and Rheumatoid Arthritis. J Periodontol 2006; 77: 280-8.

Loos BG, Roos MTL, Schellekens PTA, Velden U van der, Miedema F. Lymphocyte Numbers and Function in Relation to Periodontitis and Smoking. $J$ Periodontol 2004; 75: 557-64.

53 Kalburgi V, Sravya L, Warad S, Vijayalaxmi K, Sejal P, Hazeil D. Role of systemic markers in periodontal diseases: A possible inflammatory burden and risk factor for cardiovascular diseases? Ann Med Health Sci Res 2014; 4: 388.

54 Wohlfeil M, Scharf S, Siegelin Y, et al. Increased systemic elastase and C-reactive protein in aggressive periodontitis (CLOI-D-00160R2). Clin Oral Investig 2012; 16: 1199-207.

Vieira CLZ, Cury PR, Miname MH, et al. Severe Periodontitis Is Associated With Diastolic Blood Pressure Elevation in Individuals With Heterozygous Familial Hypercholesterolemia: A Pilot Study. J Periodontol 2011; 82: 683-8.

Craig RG, Yip JK, So MK, Boylan RJ, Socransky SS, Haffajee AD. Relationship of Destructive Periodontal Disease to the Acute-Phase Response. J Periodontol 2003; 74: 1007-16.

57 Furuichi $\mathrm{Y}$, Shimotsu A, Ito $\mathrm{H}$, et al. Associations of Periodontal Status with General Health Conditions and Serum Antibody Titers for Porphyromonas gingivalis and Actinobacillus actinomycetemcomitans . J Periodontol 2003; 74: 1491-7.

58 Hutter JW, Van Der Velden U, Varoufaki A, Huffels RAM, Hoek FJ, Loos BG. Lower numbers of erythrocytes and lower levels of hemoglobin in periodontitis patients compared to control subjects. J Clin Periodontol 2001; 28: 930-6.

59 Loos BG, Craandijk J, Hoek FJ, Dillen PMEW, Velden U Van Der. Elevation of Systemic Markers Related to Cardiovascular Diseases in the Peripheral Blood of Periodontitis Patients. J Periodontol 2000; 71: 1528-34.

Kolte R, Kolte A, Deshpande N. Assessment and comparison of anemia of chronic disease in healthy subjects and chronic periodontitis patients: A clinical and hematological study. J Indian Soc Periodontol 2014; 18: 183. 
Thomas B, Gautam A, Prasad Br, Kumari S. Evaluation of micronutrient (zinc, copper and iron) levels in periodontitis patients with and without diabetes mellitus type 2: A biochemical study. Indian J Dent Res 2013; 24: 468.

Al-Rasheed A. Elevation of white blood cells and platelet counts in patients having chronic periodontitis. Saudi Dent J 2012; 24: 17-21.

Prakash S, Dhingra K, Priya S. Similar hematological and biochemical parameters among periodontitis and control group subjects. Eur J Dent 2012; 6: 287-94.

64 Ustaoglu G, Erdal E, İnanır M. Does periodontitis affect mean platelet volume(MPV) and plateletcrit (PCT) levels in healthy adults? Rev Assoc Med Bras 2020; 66: 133-8.

Acharya AB, Shetty IP, Jain S, et al. Neutrophil-to-lymphocyte ratio and platelet-to-lymphocyte ratio in chronic periodontitis before and after nonsurgical therapy. J Indian Soc Periodontol 2019; 23: 419-23.

66 Torrungruang K, Ongphiphadhanakul B, Jitpakdeebordin S, Sarujikumjornwatana S. Mediation analysis of systemic inflammation on the association between periodontitis and glycaemic status. J Clin Periodontol 2018; 45: 548-56.

Lobão WJM, Carvalho RCC DE, Leite SAM, et al. Relationship between periodontal outcomes and serum biomarkers changes after non-surgical periodontal therapy. An Acad Bras Cienc 2019; 91: 1-10.

68 Ramich T, Asendorf A, Nickles K, et al. Inflammatory serum markers up to 5 years after comprehensive periodontal therapy of aggressive and chronic periodontitis. Clin Oral Investig 2018; 22: 3079-89.

69 Wang X, Meng HX, Xu L, Chen Z, Shi D, Lv D. Mean platelet volume as an inflammatory marker in patients with severe periodontitis. Platelets 2015; 26: 67-71.

70 Leite ACE, de Araújo Carneiro VM, do Carmo Machado Guimarães M. Efeitos da terapia periodontal sobre proteína C-reativa e HDL no soro de indivíduos com periodontite. Brazilian J Cardiovasc Surg 2014; 29: 69-77.

71 Eickholz $\mathrm{P}$, Siegelin $\mathrm{Y}$, Scharf $\mathrm{S}$, et al. Non-surgical periodontal therapy decreases serum elastase levels in aggressive but not in chronic periodontitis. J Clin Periodontol 2013; 40: 327 33.

Siqueira MA de S, Fischer RG, Pereira NR, et al. Effects of non-surgical periodontal treatment on the L-arginine-nitric oxide pathway and oxidative status in platelets. Exp Biol Med 2013; 238: 713-22.

73 Devanoorkar A, Dwarakanatha CD, Gundanavara G, Kathariyab R, Patilc SR. Evaluation of serum resistin levels in periodontal health and disease and effects of non surgical periodontal therapy on its levels. Dis Markers 2012; 32: 289-94.

74 Marcaccini AM, Meschiari CA, Sorgi CA, et al. Circulating Interleukin-6 and High-Sensitivity CReactive Protein Decrease After Periodontal Therapy in Otherwise Healthy Subjects. $J$ Periodontol 2009; 80: 594-602.

75 Stein JM, MacHulla HKG, Smeets R, Lampert F, Reichert S. Human leukocyte antigen polymorphism in chronic and aggressive periodontitis among Caucasians: A meta-analysis. $J$ Clin Periodontol 2008; 35: 183-92.

76 Cafferata EA, Jerez A, Vernal R, Monasterio G, Pandis N, Faggion CM. The therapeutic potential of regulatory $T$ lymphocytes in periodontitis: A systematic review. $J$ Periodontal Res 2019; 54: 207-17.

77 Wu D, Lin Z, Zhang S, Cao F, Liang D, Zhou X. Decreased Hemoglobin Concentration and Iron Metabolism Disorder in Periodontitis: Systematic Review and Meta-Analysis. Front Physiol 2020; 10: 1-11.

78 Angkananard T, Anothaisintawee T, McEvoy M, Attia J, Thakkinstian A. Neutrophil Lymphocyte Ratio and Cardiovascular Disease Risk: A Systematic Review and Meta-Analysis. Biomed Res Int 2018; 2018. DOI:10.1155/2018/2703518. 
diabetes: Systematic review and meta-analysis of cross-sectional and prospective studies. PLoS One 2010; 5. DOI:10.1371/journal.pone.0013405.

80 Lee C Do, Folsom AR, Nieto FJ, Chambless LE, Shahar E, Wolfe DA. White blood cell count and incidence of coronary heart disease and ischemic stroke and mortality from cardiovascular disease in African-American and White men and women: Atherosclerosis Risk in Communities Study. Am J Epidemiol 2001; 154: 758-64.

81 Tong PC, Lee KF, So WY, et al. White Blood Cell Count Is Associated with Macro- and Microvascular Complications in Chinese Patients with Type 2 Diabetes. Diabetes Care 2004; 27: 216-22.

82 Ruparelia N, Chai JT, Fisher EA, Choudhury RP. Inflammatory processes in cardiovascular disease: A route to targeted therapies. Nat Rev Cardiol 2017; 14: 133-44.

83 Donath MY. Targeting inflammation in the treatment of type 2 diabetes: Time to start. Nat Rev Drug Discov 2014; 13: 465-76.

84 Tonetti MS, D'Aiuto F, Nibali L. Treatment of periodontitis and endothelial function. N Engl J Med 2007; 356: 911-20.

85 Libby P. Inflammation in atherosclerosis. Arterioscler Thromb Vasc Biol 2012; 32: 2045-51.

86 Wong BW, Meredith A, Lin D, McManus BM. The Biological Role of Inflammation in Atherosclerosis. Can J Cardiol 2012; 28: 631-41.

87 Fagundes NCF, Almeida APCPSC, Vilhena KFB, Magno MB, Maia LC, Lima RR. Periodontitis as a risk factor for stroke: A systematic review and meta-analysis. Vasc Health Risk Manag 2019; 15: 519-32.

88 Leira Y, Seoane J, Blanco M, et al. Association between periodontitis and ischemic stroke: a systematic review and meta-analysis. Eur J Epidemiol 2017; 32: 43-53.

89 D'Aiuto F, Gkranias N, Bhowruth D, et al. Systemic effects of periodontitis treatment in patients with type 2 diabetes: a 12 month, single-centre, investigator-masked, randomised trial. Lancet Diabetes Endocrinol 2018; 6: 954-65.

90 Ryder Ml. Comparison of neutrophil functions in aggressive and chronic periodontitis. Periodontol 2000 2010; 53: 124-37.

91 Aboodi GM, Goldberg MB, Glogauer M. Refractory Periodontitis Population Characterized by a Hyperactive Oral Neutrophil Phenotype. J Periodontol 2011; 82: 726-33.

92 Hirschfeld J. Dynamic interactions of neutrophils and biofilms. J Oral Microbiol 2014; 6: 1-10.

93 Belkaid Y, Hand TW. Role of the microbiota in immunity and inflammation. Cell 2014; 157 : $121-41$.

94 Poggiali E, Migone De Amicis M, Motta I. Anemia of chronic disease: A unique defect of iron recycling for many different chronic diseases. Eur J Intern Med 2014; 25: $12-7$.

95 Madu AJ, Ughasoro MD. Anaemia of Chronic Disease: An In-Depth Review. Med Princ Pract 2017; 26: 1-9.

96 Straat M, Van Bruggen R, De Korte D, Juffermans NP. Red blood cell clearance in inflammation. Transfus Med Hemotherapy 2012; 39: 353-60.

97 Catharine Ross A. Impact of chronic and acute inflammation on extra- and intracellular iron homeostasis. Am J Clin Nutr 2017; 106: 1581S-1587S.

98 Romandini M, Laforí A, Romandini P, Baima G, Cordaro M. Periodontitis and platelet count: A new potential link with cardiovascular and other systemic inflammatory diseases. J Clin Periodontol 2018; 45: 1299-310.

99 Hsu HC, Tsai WH, Jiang ML, et al. Circulating levels of thrombopoietic and inflammatory cytokines in patients with clonal and reactive thrombocytosis. J Lab Clin Med 1999; 134: 392_ 7.

100 Kaser A, Brandacher G, Steurer W, et al. Interleukin-6 stimulates thrombopoiesis through thrombopoietin: Role in inflammatory thrombocytosis. Blood 2001; 98: 2720-5. 
101 Kaushansky K. Determinants of platelet number and regulation of thrombopoiesis. Hematology 2009; : 147-52.

102 Kapur R, Zufferey A, Boilard E, Semple JW. Nouvelle Cuisine: Platelets Served with Inflammation. J Immunol 2015; 194: 5579-87.

103 Semple JW, Italiano JE, Freedman J. Platelets and the immune continuum. Nat Rev Immunol 2011; 11: 264-74.

104 Sreeramkumar V, Adrover J, Ballesteros I, et al. Neutrophils scan for activated platelets to initiate inflammation. Science (80- ) 2014; 346: 1234-8.

Figure and Table legends

Figure 1: PRISMA Flow diagram.

Figure 2: White Blood Cells count (WBC) (10\%/L) of No Periodontitis versus Periodontitis individuals. Subgroup analysis according to the presence of chronic or aggressive periodontitis.

Figure 3: White Blood Cells count (WBC) (10\%/L) of baseline values (control) versus follow-up of CP patients after Nonsurgical Periodontal Treatment.

Table 1: Summary of quantitative estimates of meta-analyses when comparing hematologic changes in patients with periodontitis versus controls.

Table 2: Summary of quantitative estimates of meta-analyses when comparing hematologic changes in patients with chronic periodontitis versus controls.

Table 3: Summary of quantitative estimates of meta-analyses when comparing hematologic changes in patients with aggressive periodontitis versus controls.

Table 4: Summary of quantitative estimates of meta-analyses when comparing non-severe versus severe chronic periodontitis leukogram levels, and the influence of nonsurgical periodontal treatment efficacy on white blood cells levels. 